\title{
Physicochemical characteristics of garlic (Allium sativum L.) oil: Effect of extraction procedure
}

\author{
Ali Rafe ${ }^{1, *}$, Mohsen Saberi Nadjafi ${ }^{2}$ \\ ${ }^{1}$ Department of Food Nanotechnology, Research Institute of Food Science and Technology (RIFST), Mashhad, Iran \\ ${ }^{2}$ Industrial Biotechnology Institute, Iranian Academic Center for Education, Culture and Research (ACECR), Mashhad, Iran \\ Email address: \\ rafe@rifst.ac.ir (A. Rafe)
}

To cite this article:

Ali Rafe, Mohsen Saberi Nadjafi. Physicochemical Characteristics of Garlic (Allium sativum L.) Oil: Effect of Extraction Procedure. International Journal of Nutrition and Food Sciences. Special Issue: Optimizing Quality and Food Process Assessment.

Vol. 3, No. 6-1, 2014, pp. 1-5. doi: 10.11648/j.ijnfs.s.2014030601.11

\begin{abstract}
Garlic oil (GO) is behaves as a nutraceutical compound; however, its application is limited due to its pungency, undesirable aroma, low stability and solubility. The physicochemical properties of GO are certainly key parameters in the encapsulation process. Therefore, the aim of the current work was to study the effect of different extraction procedures such as solvent extraction, distillation method and supercritical fluid extraction (SCF) on the physicochemical properties of garlic oil. The yield of GO was influenced by extraction method and was approximately $5.5,6$ and $7 \%$ for steam distillation, solvent methods and $\mathrm{SCF}-\mathrm{Co}_{2}$, respectively. The specific gravity was obtained as $0.894 \mathrm{~g} / \mathrm{cm}^{3}$ and independent to the extraction method, while the viscosity was changed and more viscosity was obtained by $\mathrm{SCF}-\mathrm{Co}_{2}$. The chemical analyses revealed 2.5 as the acid value, 1.27 for free fatty acids, 1.8 as the saponificacation value and 14.5 as the iodine value of GO by SCF- $-\mathrm{Co}_{2}$. The results showed that the SCF- $-\mathrm{Co}_{2}$ had minor quality and nutritional loss on the GO, and could be applied for special purposes such as encapsulation of garlic oil.
\end{abstract}

Keywords: Garlic Oil, Extraction, Supercritical Fluid, Extraction Yield, Viscosity

\section{Introduction}

Allium sativum L., which is commonly known as garlic, belongs to the onion family Alliaceae and is closely related to the onion, shallot, leek, chive, and rakkyo [1]. It has been used throughout the world for both culinary and medicinal purposes [2] and its world production was about 25 million metric tons in 2012 [3]. It has a pungent and spicy flavor, which is mainly related to sulfur containing compounds such as allicin, alliin, ajoene, dially disulfide, dithiin and s-allylcysteine. It is believed that dially disulfide is an important odour component in the garlic. Allicin is the component responsible for the spiciness in the raw garlic and has powerful antibiotic and antifungal properties. It derives from the precursor alliin in fresh garlic tissue through the enzymatic action of alliinase. Allicin and other degradation products from alliin also give garlic a characteristic flavor [4].

Garlic essential oil is extracted by a process of steam distillation of the garlic cloves using solvents such as n-hexane or petroleum ether. Garlic oil (GO) contains a variety of sulfide such as dially disulfide and dilly trisulfide. Due to the pungency odour of garlic essential oil, the commercial GO capsules generally contain vegetable oil and a small amount of GO. Other garlic supplements are dehydrated garlic powder; garlic oil macerate and aged garlic extract [5]. Garlic oil behaves as a nutraceutical compound, with numerous applications in food and pharmaceutical industries such as the flavouring of some cuisine such as salads, and sauces; can reduce blood pressure, prevent cancer and cardiovascular diseases through reducing serum LDL cholesterol and triglyceride $[6,7]$. In addition, there are many reports on the antimicrobial, antioxidant, antithrombotic, and anticancer properties of garlic oil, which could be used to prevent nausea, diarrhea, ease coughs, and treatment in conditions such as malaria and cholera $[6,8,9]$.

Supercritical fluids (SCFs) often prove to be efficient solvents with better transport properties (diffusivity, mass transfer coefficient, penetration ability) than most common organic solvents in the liquid state [10]. When using carbon dioxide $\left(\mathrm{Co}_{2}\right)$ in particular, a high selectivity for complete separation of solvent traces from the extract and treated 
matrix can be achieved [11]. A further quality advantage for oxidation-prone substances is that these are exposed to neither oxygen nor high temperatures during treatment with supercritical $\mathrm{Co}_{2}$, in contrast to conventional separation methods. Thus, "premium" quality products can be obtained by SCF extraction with $\mathrm{Co}_{2}$.

There have been some reports in the scientific literature on the SCF extraction of allium species, and although many of these correspond to analytical applications for garlic [12-14], there are also reports on process development for industrial applications for other allium species. There were no compositional differences between extracts of a homogenate of fresh garlic in water using $\mathrm{Co}_{2}$ and organic solvents [12].

The physicochemical properties of GO such as viscosity can be useful in understanding its role on food and pharmaceutical products, as well as providing suitable knowledge to encapsulate garlic oil with a proper carrier or coating material. Due to lack of knowledge in physicochemical properties of GO, the main objectives of the current work was determining these properties of GO and the effect of extraction procedure such as steam distillation, solvent extraction method and SCF extraction with $\mathrm{Co}_{2}$ on its chemical and physicochemical characteristics.

\section{Materials and Methods}

\subsection{Materials}

The garlic cloves were obtained from farmland of Hamedan (Hamedan, $34^{\circ} 48^{\prime} \mathrm{N} 48^{\circ} 31^{\prime} \mathrm{E}$, Iran). The transparent shell of garlic samples were removed manually and were sliced into small pieces. The sliced garlic samples were dehydrated overnight in a vacuum oven (Heraeus, VT $6130 \mathrm{M}$, Germany) at $60^{\circ} \mathrm{C}$ and one bar vacuum pressure. Then, dried samples were packed in polystyrene bags and maintained in a refrigerator at $4^{\circ} \mathrm{C}$ for further uses. All reagents were of analytical grade. All the solutions and dilutions were carried out using distilled deionized water. The chemical and physiochemical analyses were performed in triplicates.

\subsection{Garlic Oil Extraction}

The solvent extraction method was compared with supercritical $\mathrm{Co}_{2}$ extraction. The solvent extraction was carried out by a soxhlet extractor using commercial hexane for four hours. The oils were obtained after the solvent was removed by a rotary evaporator (B-480, BUCHI Switzerland) under reduced temperature $\left(60^{\circ} \mathrm{C}\right)$ and pressure to remove any excess solvent. The extracted garlic oil was stored in a refrigerator at $4^{\circ} \mathrm{C}$ for subsequent experiments.

The supercritical $\mathrm{Co}_{2}$ extraction was carried out in an SFE-2.5L process development unit, which was made by the Research Institute of Food Science and Technology (RIFST) experts (Fig 1). The apparatus was similar to previous designs, used for supercritical extraction $[15,16]$. The extraction vessel was equipped with a heating jacket and temperature was controlled by recirculation of heated water through a thermally equilibrated bath. The experiments were carried out in the dynamic mode using $800 \mathrm{~g}$ samples and $20 \mathrm{~g} / \mathrm{min}$ of $\mathrm{Co}_{2}$. Some preliminary experiments were carried out to select the extraction time and the particle size. The extraction was performed at optimum temperature and pressure $\left(50^{\circ} \mathrm{C}, 100\right.$ bar), which was mentioned in previous works [16].

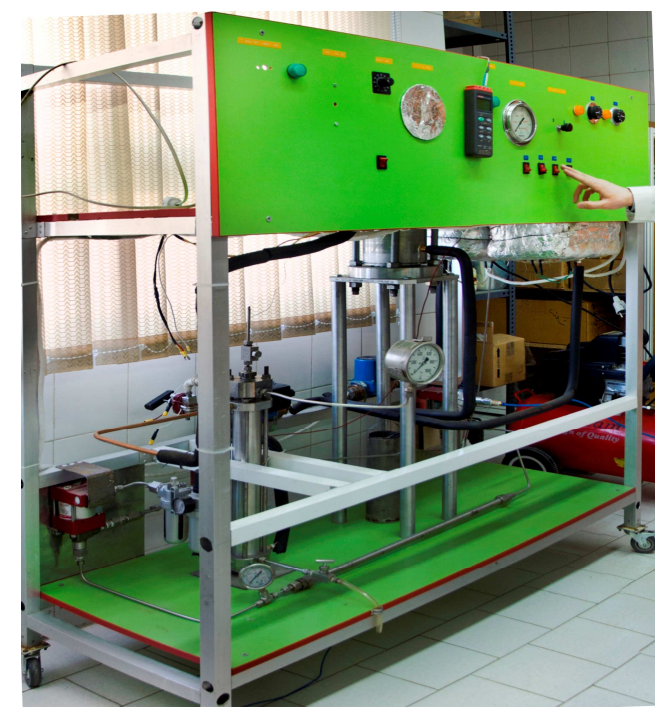

Figure 1. The supercritical $\mathrm{Co}_{2}$ extractor.

\subsection{Extraction Efficiency}

The garlic oil extracted by solvent method was recovered by complete distilling of the solvent on a heating mantle. Then, it was transferred into a measuring cylinder and placed over a water bath for complete evaporation of solvent for about 2-3 hours. The volume of oil was expressed as extraction yield (\%) as follows:

\section{Extraction yield $(\%)=($ Volume of the oil $) /($ Weight of sample) $* 100$}

The extraction efficiency of SFE and steam distillation methods were determined similarly.

\subsection{Physicochemical Properties}

The chemical properties of GO samples, which included free fatty acids (FFA), peroxide, iodine value (IV) and saponification values were determined by the AOAC method [17]. The density and specific gravity of GO were determined at $25^{\circ} \mathrm{C}$ by a $10 \mathrm{ml}$ pycnometer (Gay-Lussac, Germany) [18]. The refractive index was measured at $25^{\circ} \mathrm{C}$ by a digital refractometer with four digits (RX5000- $\alpha$, Atago, Japan).

The rheological properties of GO was measured using a rotational viscometer (Model DV-III ULTRA, Brookfield Engineering, Inc., USA) at $25^{\circ} \mathrm{C}$ using the spindle SC4-18 at shear rates from 10 to $300 \mathrm{~S}^{-1}$. Samples were placed in 
the temperature-controlled measurement vessel and allowed to equilibrate to the required temperature for $5 \mathrm{~min}$ prior the experiments. The volumes of sample as well as the immersion depth of spindle were kept constant throughout the experiments. Measurements were performed in triplicate with $10 \mathrm{~s}$ intervals and the data were averaged. The viscosity was determined by taking the slope of shear stress-shear rate curve at different temperatures $(25,40$ and $\left.60^{\circ} \mathrm{C}\right)$.

\section{Results and Discussion}

\subsection{Yield Extraction of Garlic Oil}

The extraction method of garlic oil has significant effect on yield extraction. The yield of GO by steam distillation was approximately $5.5 \%$, while yield extraction of GO by SCF- $-\mathrm{Co}_{2}$ and solvent method were 7 and $6 \%$, respectively. The particle size and shape of samples in extraction process is a vital factor in yield and efficiency. It was reported that yield extraction of onion powder and garlic flake with ethanol was about 35 and 5.5\%, respectively [16]. Therefore, it can be found that the percentage yield of GO by $\mathrm{SCF}-\mathrm{Co}_{2}$ is more than the other common methods, and can compensate the installation charges of SCF in the commercial scale-up. Although, the yield efficiency of GO is lower than many commercial oils such as soybean with at least $20 \%$ efficiency, however it should be mentioned that GO has special properties which make it profitable for industrial applications.

Another factor that may have affected differences in yield between garlic and onion is sample pretreatment. In our work there was a problem of agglomeration of milled garlic flakes during large-scale extraction, which was not reported for the extraction of onion powder and may have limited the yield of the process. As a result of agglomeration in a packed bed, the specific surface for extraction diminishes and the diffusion path within the solid matrix increases, creating mass transfer limitations to yield. The extraction yield of garlic flakes with $\mathrm{SCF}-\mathrm{Co}_{2}$ changed with sample particle size and process temperature, pressure, and time. Since the milled substrate showed a tendency to agglomerate during extraction, optimal particle size was determined as 1.18 to $4.75 \mathrm{~mm}$.

\subsection{Physicochemical Properties}

The physicochemical properties of garlic oil, which was obtained by different extraction procedures, are presented in Table 1. The specific gravity of GO by solvent method was $0.894 \mathrm{~g} / \mathrm{cm} 3$, which is lower than that of the most edible oils such as sunflower, cotton seed and olive oil (about $0.919 \mathrm{~g} / \mathrm{cm} 3$ ) [19-21]. In addition, there was no statistical difference in specific gravity of GO in different extraction methods (Table 1). This amount of specific gravity indicates the feasibility of GO production at a commercial scale. However, the colour of the oil was light yellow and had a pungent odour in the steam distillation and solvent extraction method, but its colour changed to greenish yellow in the SCF procedure, which may be attributed to penetration of garlic germs to the oil. The melting point of garlic oil $\left(\sim 12^{\circ} \mathrm{C}\right)$ was also lower than that of most edible oils and liquids, which is attributed to the high amount of unsaturated fatty acids such as linoleic acid and linolenic acid that have valuable nutritional properties.

The refractive index can be considered as an oil trait, which depends on saturation degree of oil and the ratio of cis to trans bonds in oil [25]. Since, the refractive index of garlic oil was about 1.43340 and less than many of edible oils such as sunflower oil (1.4663), palm (1.4531) and soybean oil (1.4675); it can be used for differentiate it from other edible oils.

Since, it has a high amount of unsaturated fatty acids, it is more prone to rancidity and needs to be prevented from oxidation. Thus, the oxidation deterioration of oil was measured by \% FFA, acid value and peroxide value. Although, the peroxide value of GO (2\%) was lower than that of other edible oils such as palm kernel oil (3.58\%), but due to its high content of unsaturated fatty acids, it cannot be concluded that GO can be kept for a very long period of time [22]. In addition, the peroxide value changed according to the extraction procedure and it was less than $1 \%$ in SCF extraction in comparison with solvent and steam distillation method (Table 1). Therefore, it is observed that the SCF extraction with $\mathrm{Co}_{2}$ has minor quality loss on the GO.

The acid value and percent of FFA in SFC extraction were less than those obtained in the solvent and steam distillation method (Table 1), thus concluding that the efficiency of this method enables less chemical damage to the oil. As the acid value of oil was higher, the storage quality was lower [23]. Thus, the results showed that the garlic oil had a proper storage quality when compared with oil containing a high amount of unsaturated fatty acids [24]. Since, the FFA of GO was about $1.4 \pm 0.05 \%$, it has the potential to be used as edible oil and can thus be produced industrially.

The saponification value of the garlic oil was found to be $190.74 \mathrm{mgKOH} / \mathrm{g}$, which is higher than that of some oils such as shear butter oil (183.1), castor seed oil (176-187), and canola oil (168-181), while the saponification value is lower than that of most of edible oils such as palm, palm kernel, sunflower, soybean and olive oils [25].

In order to measure the amount of unsaturated fatty acids in GO, iodine value was determined. The iodine value was $11.70 \% \pm 0.05$, and is observed to be low when compared to $16.0 \mathrm{~g} / 100 \mathrm{~g}$ which is the value for Tiger nut oil [26]. The oil shows quite a degree of unsaturated fatty acid indicating that the oil is suitable for consumption and can also be used as a non drying oil, which is useful in the manufacture of soap. In addition, the IV of GO was dependent to some extend on the method of extraction. The iodine value of GO by $\mathrm{SFC}-\mathrm{Co}_{2}$ was about $14.5 \%$, which was more than the other methods of extraction. On the other hand, the SFC method had limited chemical changes on the garlic oil and 
maintained the nutritional properties of GO.

The typical curve of shear stress-shear rate of GO is shown in Fig 2. It can be observed that garlic oil showed Newtonian behavior like other edible oils [26]. The viscosity was obtained from the slope of the fit of experimental shear stress-shear rate data to the Newton's law of viscosity equation (Eq. 1). Although; its viscosity was about $70.9 \mathrm{mPas}$ at $25^{\circ} \mathrm{C}$, this is deemed to be more than the viscosities of similar edible oils. This high amount of viscosity was attributed to the specific chemical compounds such as disulfides in garlic oil. In addition, the viscosity of GO was influenced by the extraction methods and the lowest viscosity was obtained for steam distillation and solvent method, respectively.

\section{$\tau=\mu \gamma$}

where $\tau$ is shear stress $(\mathrm{mPa}), \gamma$ is shear rate $\left(\mathrm{s}^{-1}\right)$ and $\mu$ is viscosity (mPa.s).

Table 1. Physicochemical properties of Garlic oil extracted by different methods

\begin{tabular}{llll}
\hline Parameters & Solvent Extraction & Steam distillation & SFC-CO $_{2}$ \\
\hline Yield extraction, \% & 6 & 5.5 & 7 \\
Oil color & Light yellow & Light yellow & Light green \\
Odor & Pungent & Pungent & Pungent \\
SG & $0.894 \pm 0.001$ & $0.897 \pm 0.001$ & $0.896 \pm 0.001$ \\
Melting point & $12 \pm 0.5$ & $12 \pm 0.5$ & \pm 0.5 \\
Refractive index & $1.43340 \pm 0.0001$ & $1.43344 \pm 0.0001$ & $1.43443 \pm 0.0001$ \\
Viscosity, $\mathrm{mPas}$ & $64.20 \pm 1.4$ & $68.42 \pm 2.1$ & $70.8 \pm 1.5$ \\
Acid value, $\mathrm{mgKOH} / \mathrm{g}$ & $2.80 \pm 0.20$ & $2.70 \pm 0.20$ & $2.50 \pm 0.15$ \\
FFA, \% & $1.41 \pm 0.28$ & $1.60 \pm 0.32$ & $1.27 \pm 0.14$ \\
Saponification value, $\mathrm{mgKOH} / \mathrm{g}$ & $190.74 \pm 1.05$ & $192.04 \pm 1.25$ & $196.51 \pm 0.85$ \\
Peroxide value, $\mathrm{mg} / \mathrm{l} 00 \mathrm{~g}$ & $2.0 \pm 0.20$ & $1.80 \pm 0.20$ & $1.8 \pm 0.10$ \\
Iodine value, $\mathrm{I}_{2 \mathrm{~g} / 100 \mathrm{~g}}$ & $11.7 \pm 0.20$ & $11.5 \pm 0.10$ & $14.5 \pm 0.10$ \\
\hline
\end{tabular}

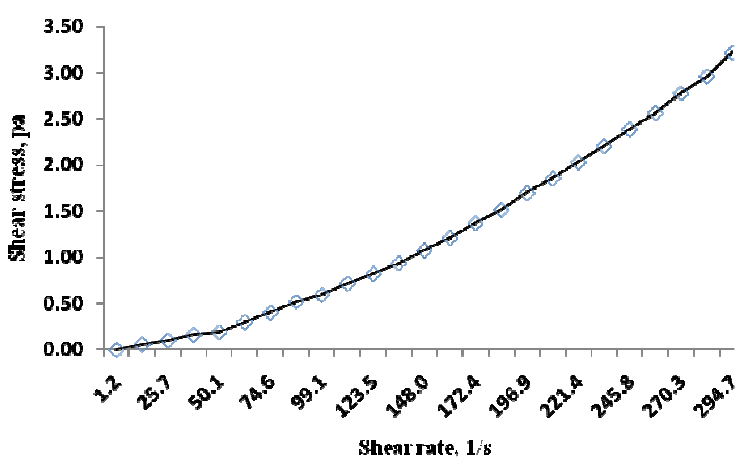

Figure 2. Typical curve of shear stress-shear rate of GO.

\section{Conclusion}

The physicochemical properties of garlic oil are critical parameters in the encapsulation process and depend on the extraction methods. Yield extraction of $\mathrm{GO}$ by $\mathrm{SCF} \mathrm{Co}_{2}$ was more than that of other methods and thus has to be considered in its production. The specific gravity of GO by solvent method was similar to many edible oils and there were no statistical differences in specific gravity of GO in the other extraction methods. The specific gravity indicates the feasibility of GO production at a commercial scale. The melting point of garlic oil was also lower than that of most of edible oils, which certainly related to the high content of unsaturated fatty acids such as linoleic acid and linolenic acid that have valuable nutritional properties. The refractive index of garlic oil can be used for its identification form other edible oil sources. The chemical results showed that quality garlic oil can be maintained by $\mathrm{SCF}-\mathrm{Co}_{2}$ for special purposes such as encapsulation. In addition, high viscosity of garlic oil makes it proper for encapsulation. In fact, the $\mathrm{SFC}-\mathrm{Co}_{2}$ is the proper procedure for GO extraction, if it would be applied in encapsulation process. Garlic oil encapsulation based on the polymer blending laws is being investigated by our researchers and will be presented at a later stage.

\section{References}

[1] Block, E. (2010). Garlic and other Alliums: The lore and the science. Royal society of Chemistry. pp. 190-9.

[2] McGee, H. (2004). The Onion Family: Onions, Garlic, Leeks. On food and cooking revised edition sceribnes. pp. 310-313.

[3] faostat.fao.org/site/339/default.aspx

[4] Block, E. (1992). The organosulfur chemistry of the genus Allium- implications for organic sulfur chemistry. Angewandte Chemie International Edition. 140, 1158-1203.

[5] Amagase, H., Petesch, B.L., Matsuura, H., Kasuga, S., \& Hakura, Y. (2001). Intake of Garlic and its bioactive components. The Journal of nutrition. 131, 955- 962.

[6] Carson, J. F. (1987). Chemistry and Biological Properties of Onions and Garlic, Food Reviews International. 3, 71.

[7] Andreatta, A.E., Foco, G., Mabe, G., \& Bottini, S.B. (2005). Extraction of garlic oil with Quasi-Critical Solvents. ENPROMA, Argentina. 1-9.

[8] Nagpurkar, A., Peschell, J., \& Holub, B. J. (2000). Garlic constituents and disease prevention. In G. Massa, \& B. D. Oomah (Eds.), Herbs, botanicals \& teas (pp. 1-21). Lancaster, PA: Technomic Publishing Co., Inc. 
[9] Turner, J. (2004). Spices The History of a Temptation of Vintage Books .1st Ed. 16.

[10] Brunner, G. (1994). Gas extraction: an introduction to fundamentals of supercritical fluids and the application to separation processes. New York, NY: Springer.

[11] del Valle, J. M., \& Aguilera, J. M. (1989). Effects of substrate densification and $\mathrm{CO}_{2}$ conditions on supercritical extraction of mushroom oleoresins. Journal of Food Science, 54, $135-141$.

[12] Calvey, E. M.; Matusik, J. E.; White, K. D.; Betz, J. M.; Block, E.; Littlejohn, M. H.; Naganathan, S.; Putman, D. (1994). Off-line supercritical-fluid extraction of thiosulfinates from garlic and onion. J. Agr. Food Chem. 42 (6), 1335-1341.

[13] Calvey, E. M.; Matusik, J. E.; White, K. D.; DeOrazio, R.; Sha, D.; Block, E. (1997). Allium chemistry: supercritical fluid extraction and LC-APCI-MS of thiosulfinates and related compounds from homogenates of garlic, onion, and ramp. Identification in garlic and ramp and synthesis of 1-propanesulfinothioic acid S-allyl ester. J. Agric. Food Chem. 45 (11), 4406-4413.

[14] Ryback, M. E., Calvey, E. M., \& Harnly, J. M. (2004). Quantitative determination of allicin in garlic: supercritical fluid extraction and standard addition of alliin. Journal of Agricultural and Food Chemistry. 52, 682-687.

[15] del Valle, J. M., Rivera, O., Teuber, O., and Palma, M.T. (2003). Supercritical $\mathrm{CO}_{2}$ Extraction of Chilean Hop (Humulus lupulus) Ecotypes, Journal of the Science of Food and Agriculture, 83, 1349.

[16] del Valle, C. Mena \& M. Budinich. (2008). Extraction of garlic with supercritical $\mathrm{CO} 2$ and conventional organic solvents. Brazilian Journal of Chemical Engineering, 25, 3, $535-542$.
[17] AOAC International. 1995. Official Methods of Analysis of the AOAC International, 16th ed. Association of Official Analytical Chemists, Arlington, VA. USA.

[18] Kopecký, F. (1999). Physics for Students of Pharmacy I. Bratislava, UK. 184 s. (in Slovak).

[19] Hodgman, C.D. \& N.A. Lange. Handbook of Chemistry and Physics. Cleveland: Chemical Rubber Co., 1924: 312-313.

[20] Weast, R.C., et al. CRC Handbook of Chemistry and Physics. Boca Raton: CRC Press, 1988-1989: F3.

[21] Mohammed M.I., \& Hamza, Z.U. (2008). Physicochemical Properties of Oil Extracts from Sesamum Indicum L. Seeds Grown in Jigawa State-Nigeria. Journal Applied Environment Management.12, 2, 99-100.

[22] Ogbuagu, M.N. (2008). Inhibitory effect of onion and garlic extracts on the rancidity of palm and palmKernel Oils. Journal Chemistry Society of Nigeria, 33, 1, 43-44.

[23] Nielsen NS, Klein A, Jacobsen C. (2009). Effect of ingredients on oxidative stability of fish oil enriched drinking yoghurt. European Journal of Lipid Science and Technology.111:337-345.

[24] Warra, A. A., Gunu, S.Y., Jega A., \& Aisha. J.A. (2011). Soap Production from Shea nut Butter. International Journal of Applied Sciences. 5, 4:410-412.

[25] Apple white, Thomas. (1985). Bailey's industrial oil and fat products. John wiley and sons. Newyork.

[26] Kamalu, C.I.O, \& Oghome, P. (2008). Extration and Characterization of Tiger Nut oil. Journal of chemistry Society of Nigeria. 33, 1, 79-87.

[27] Fasina O.O. \& Colley Z. (2008). Vscosity and specific heat of vegetable oils as a function of temperature: $35^{\circ} \mathrm{C}$ to $180^{\circ} \mathrm{C}$. International Journal of Food Properties, 11: 738-746. 